\title{
Amplitude modulation of three-dimensional low-frequency solitary waves in a magnetized dusty superthermal plasma
}

\author{
Shalini $^{1}$ - A. P. Misra ${ }^{2}$ N. S. Saini ${ }^{1}$
}

Received: 28 January 2017/ Accepted: 17 June 2017/Published online: 30 June 2017

(C) The Author(s) 2017. This article is an open access publication

\begin{abstract}
The amplitude modulation of three-dimensional (3D) dust ion-acoustic wave (DIAW) packets is studied in a collisionless magnetized plasma with inertial positive ions, superthermal electrons and negatively charged immobile dust grains. By using the reductive perturbation technique, a 3D-nonlinear Schrödinger equation is derived, which governs the slow modulation of DIAW packets. The latter are found to be stable in the low-frequency $\left(\omega<\omega_{\mathrm{c}}\right)$ regime, whereas they are unstable for $\omega>\omega_{\mathrm{c}}$, and the modulational instability is related to the modulational obliqueness $(\theta)$. Here, $\omega\left(\omega_{\mathrm{c}}\right)$ is the nondimensional wave (ion-cyclotron) frequency. It is shown that the superthermal parameter $\kappa$, the frequency $\omega_{\mathrm{c}}$ as well as the charged dust impurity $(0<\mu<1)$ shift the MI domains around the $\omega-\theta$ plane, where $\mu$ is the ratio of electron-to-ion number densities. Furthermore, it is found that the decay rate of instability is quenched by the superthermal parameter $\kappa$ with cutoffs at lower wave number of modulation $(K)$; however, it can be higher (lower) with increasing values of $\mu\left(\omega_{\mathrm{c}}\right)$ having cutoffs at higher values of $K$.
\end{abstract}

Modulational instability $\cdot$ NLS equation · Superthermal plasma $\cdot$ Dusty plasma $\cdot$ Solitary wave

\section{N. S. Saini}

nssaini@yahoo.com

Shalini

shal.phy29@gmail.com

\section{A. P. Misra}

apmisra@gmail.com

1 Department of Physics, Guru Nanak Dev University, Amritsar, India

2 Department of Mathematics, Siksha Bhavana, Visva-Bharati University, Santiniketan, India

\section{Introduction}

The nonlinear features of solitary waves in dusty plasmas have been of great importance over the last many years due to their wide range of applications in space, astrophysical and laboratory environments [1-3]. Dust grains are typically micron or sub-micron sized particles and are ubiquitous ingredients in our universe. The presence of charged dust grains in an electron-ion plasmas not only alters the characteristics of ion-acoustic solitary waves (IASWs), but also modifies the ion-acoustic wave as well as generates a new kind of mode, namely, the dust-acoustic (DA) wave. More than three decades ago [4], reported theoretically the existence of DIA waves in a dusty plasma. Later, in laboratory experiments [5], confirmed the existence of these waves. A large number of investigations on DIA waves in multicomponent plasmas have been reported in the framework of Sagdeev's approach as well as reductive perturbation technique.

Furthermore, in many observations it has been confirmed that superthermal particles exist in space plasmas [6-9] and laboratory environments [10]. These superthermal particles are described by Lorentzian (kappa) distribution which is more appropriate for analysis of data rather than a Maxwellian distribution [9]. Furthermore, such distribution has been widely used to investigate various collective modes as well as nonlinear coherent structures like solitons, shocks, envelope solitons through the description of Korteweg-de Vries (KdV), Korteweg-de Vries Burgers (KdVB) and nonlinear Schrödinger (NLS) equations [11-18].

On the other hand, there has also been a growing interest in investigating the nonlinear modulation of electrostatic waves in plasmas owing to their importance not only in space and astrophysical environments, but also in 
laboratory plasmas. The modulational instability (MI) of nonlinear waves in plasmas has been a well-known mechanism for the localization of wave energy, which leads to the formation of bright envelope solitons. However, in the absence of instability, the evolution of the system can be in the form of dark envelope solitons. Furthermore, due to a small plane wave perturbation, MI can have exponential growth which leads to the amplification of the sidebands, and thus break up the uniform waves into a train of oscillations. A large number of investigations on MI of electrostatic or electromagnetic waves can be found in the literature [14, 18-26]. To mention few, the MI of obliquely propagating DIA waves in an unmagnetized plasma containing positive ions, electrons and immobile dust grains was reported by [19]. It was observed from the stability analysis that the obliqueness in the modulation direction has a profound effect on the condition of MI. They also observed the influence of ion temperature on the amplitude modulation of wave and noticed that wave stability profile may be strongly modified by ion temperature [21]. Furthermore, the nonlinear propagation of wave envelopes in an unmagnetized superthermal dusty plasma was investigated by El-Labany [27] et al. They showed that the electron superthermality and the dust grain charge significantly modify the profiles of the wave envelope and the associated regions of instability. Ahmadihojatabad et al. [28] studied the influence of superthermal and trapped electrons on the obliquely propagating ion-acoustic waves (IAWs) in magnetized plasmas. Bains et al. [25] addressed the MI of ion-acoustic wave envelopes in a multicomponent magnetized plasma using a quantum fluid model. They observed that the ion number density, the constant magnetic field and the quantum coupling parameter have strong effects on the growth rate of MI. Also, the nonlinear propagation of DIA wave envelopes in a three-dimensional magnetized plasma containing nonthermal electrons featuring Tsallis distribution, both positive and negative ions, and immobile charged dust was investigated by [29].

To the best of our knowledge the investigation of MI of DIAWs in a magnetized dusty plasma containing superthermal electrons has not yet been reported. Our purpose in this investigation is to consider the propagation of DIA wave envelopes in a magnetized dusty plasma containing cold positive ions and superthermal electrons. We have employed the standard multiple-scale perturbation technique to derive the NLS equation. It was shown that in earlier investigations [30] that MI of ion acoustic waves is significantly influenced by the presence of superthermal electrons and growth rate is larger in the presence of more superthermal electrons. We have, however, investigated the combined effects of the external magnetic field, dust concentration and the superthermality of electrons on the MI of DIA wave packets. It is shown that the superthermality of electrons (via $\kappa$ ), the charged dust impurity and the external magnetic field shift the MI domains around the $\omega-\theta$ plane, where $\omega$ is the wave frequency and $\theta$ stands for modulational obliqueness. Further, we have also studied the decay rate of MI by different plasma parameters.

The paper is organized as follows: in Sect. 2, the basic equations governing the nonlinear dynamics of DIA wave envelopes in magnetized superthermal plasmas are presented and the three-dimensional NLS equation is derived. The effects of various physical parameters on the existence of stable/unstable regions for the modulation of DIA waves are investigated in Sect. 3. Finally, Sect. 4 contains the summary and conclusions of our results.

\section{The model equations and derivation of the 3D- NLSE}

We consider the nonlinear propagation of DIA waves in a magnetized plasma consisting of superthermal electrons, cold positive ions and negatively charged immobile dust grains. The plasma is immersed in the constant magnetic field $\mathbf{B}_{0}=B_{0} \hat{z}$. We adopt a fluid model for the dynamics of DIA waves in a magnetized plasma which consists of the continuity, momentum and the Poisson's equations. Thus, we have

$$
\begin{aligned}
& \frac{\partial n}{\partial t}+\nabla \cdot(n \mathbf{U})=0, \\
& \frac{\partial \mathbf{U}}{\partial t}+(\mathbf{U} \cdot \nabla) \mathbf{U}=-\frac{e}{M} \nabla \phi+\frac{e B_{0}}{M}(\mathbf{U} \times \hat{z}), \\
& \nabla^{2} \phi=4 \pi e\left(n_{\mathrm{e}}-n+Z_{\mathrm{d}} n_{\mathrm{d}} 0\right)
\end{aligned}
$$

where the superthermal electrons are given by the kappa distribution [31]

$$
n_{\mathrm{e}}=n_{\mathrm{e} 0}\left[1-\frac{e \phi}{(\kappa-3 / 2) k_{\mathrm{B}} T_{\mathrm{e}}}\right]^{-\kappa+1 / 2} .
$$

The set of fluid Eqs. (1)-(3) in nondimensional forms are written as

$$
\begin{aligned}
& \frac{\partial n}{\partial t}+\nabla \cdot(n \mathbf{U})=0, \\
& \frac{\partial \mathbf{U}}{\partial t}+(\mathbf{U} \cdot \nabla) \mathbf{U}=\nabla \phi+\omega_{\mathrm{c}}(\mathbf{U} \times \hat{z}), \\
& \nabla^{2} \phi=n_{\mathrm{e}}-n+(1-\mu),
\end{aligned}
$$

where $n_{\mathrm{e}}$ and $n$ are the number densities of electrons and ions normalized by the equilibrium number density of ions $n_{0}, \phi$ is the electric potential normalized by $k_{\mathrm{B}} T_{\mathrm{e}} / e, \mathbf{U} \equiv$ $(u, v, w)$ is the ion fluid velocity normalized by the DIA speed $C_{\mathrm{s}}\left(=\sqrt{k_{\mathrm{B}} T_{\mathrm{e}} / M}\right)$. The space and time coordinates 
are normalized by the Debye length $\lambda_{\mathrm{D}}[=$ $\left.\left(k_{\mathrm{B}} T_{\mathrm{e}} / 4 \pi n_{0} e^{2}\right)^{1 / 2}\right]$ and the inverse of ion plasma frequency $\omega_{p i}\left[=\left(4 \pi e^{2} n_{0} / M\right)^{1 / 2}\right]$, respectively. Furthermore, $\omega_{\mathrm{c}}=$ $e B_{0} / c M$ is the ion gyrofrequency normalized by $\omega_{p i}$. The charge neutrality condition yields $1-\mu=Z_{\mathrm{d}} n_{\mathrm{d} 0} / n_{0}$, where $\mu=n_{\mathrm{e} 0} / n_{0}$ is the ratio of equilibrium number densities of electrons and ions. Next, in the small-amplitude perturbations, i.e., $|\phi| \ll 1$, and, in particular, $|\phi /(\kappa-3 / 2)| \ll 1$, Eq. (4) reduces to

$n_{\mathrm{e}} \approx \mu+q_{1} \phi+q_{2} \phi^{2}+q_{3} \phi^{3}$,

where the coefficients are given by

$q_{1}=\mu \frac{(\kappa-1 / 2)}{(\kappa-3 / 2)}, q_{2}=\mu \frac{\left(\kappa^{2}-1 / 4\right)}{2(\kappa-3 / 2)^{2}}$,

$q_{3}=\mu \frac{\left(\kappa^{2}-1 / 4\right)(\kappa+3 / 2)}{6(\kappa-3 / 2)^{3}}$.

In order to derive the evolution equation for weakly nonlinear DIA wave envelopes, we employ the standard multiple-scale technique $[32,33]$ in which the coordinates are stretched as

$\xi=\epsilon x, \eta=\epsilon y, \zeta=\epsilon\left(z-V_{\mathrm{g}} t\right), \tau=\epsilon^{2} t$.

Consider $A \equiv(n, w, \phi)$ and $B \equiv(u, v)$ as the state vectors which describe the state at a position $z$ and time $t$. The perturbations from the equilibrium state $A^{(0)}=(1,0,0)^{T}$ and $B^{(0)}=(0,0)^{T^{\prime}}$ are considered by assuming $A=A^{(0)}+$ $\sum_{m=1}^{\infty} \epsilon^{m} A^{(m)}$ and $B=B^{(0)}+\sum_{m=1}^{\infty} \epsilon^{m+1} B^{(m)}$. The slowscale dependence of all perturbed state enter via the $l$-th harmonic amplitude $A_{l}^{(m)}$ and $B_{l}^{(m)}$ given as $A^{(m)}=$ $\sum_{l=-m}^{m} A_{l}^{(m)}(\xi, \eta, \zeta, \tau) e^{i l(k z-\omega t)} \quad$ and $\quad B^{(m)}=\sum_{l=-m}^{m} B_{l}^{(m)}$ $(\xi, \eta, \zeta, \tau) e^{i l(k z-\omega t)}$, where $\omega$ and $k$, respectively, represent the carrier wave frequency and the wavenumber. In order that $n, \mathbf{U}, \phi$, etc. are all real, the state variables must satisfy the reality condition with respect to its complex conjugate parts. One should note that the transverse (to the magnetic field) velocity components $u$ and $v$ appear at higher order in $\epsilon$ than the parallel component $w$. The anisotropy and higher order effects are introduced via strong magnetic field and gyro-motion of fluid, respectively, in the presence of weak perturbations [34].

We substitute the stretched coordinates (10) and the expansions given above into Eqs. (5)-(8), and collect terms in different powers of $\epsilon$ to obtain a set of reduced equations. Thus, equating the coefficients for $m=1$ and $l=1$, we obtain the following first-order quantities in terms of $\phi_{1}^{(1)}$ $n_{1}^{(1)}=\frac{k^{2}}{\omega^{2}} \phi_{1}^{(1)}$

$w_{1}^{(1)}=\frac{k}{\omega} \phi_{1}^{(1)}$,

together with the linear dispersion relation

$\omega^{2}=\frac{k^{2}}{k^{2}+q_{1}}$.

From the second-order reduced equations $(m=2, l=1)$, the following compatibility condition in terms of the group velocity of waves is obtained as

$V_{\mathrm{g}} \equiv \frac{\partial \omega}{\partial k}=q_{1} \frac{\omega^{3}}{k^{3}}$.

We have depicted the variation of the carrier wave frequency (Eq. 13) and the group velocity (Eq. 14) against the carrier wave number $k$ in Fig. 1. From the upper panel, we find that as $k$ increases, the frequency $\omega$ increases and it approaches a constant value at higher $k(>1)$. Furthermore, $\omega$ (normalized by the ion plasma frequency $\omega_{p i}$ ) increases and approaches a constant value (close to 1) as the wave number $k$ increases. Also, the value of $\omega$ increases with increasing values of the spectral index $\kappa$ (i.e., when the superthermality of electrons is somewhat relaxed); however, it remains almost unaltered for $\kappa>8$. Nevertheless, a reduction of the wave frequency is noticed with increasing values of the electron-to-ion number density ratio $\mu$. This implies that as the number density of electrons increases, more electrons will flow out of the dust grains, i.e., dust charge number decreases in order to maintain the quasineutrality. Further increase of $\mu$ may eventually lead to the case similar to the dust-free electronion plasma. Thus, negatively charged dust impurity in the plasma with $n_{0}>n_{\mathrm{e} 0}$ effectively increases the wave frequency. Such dust impurity has also a significant effect on the group velocity of waves as shown in the lower panel of Fig. 1 (see the solid and dashed lines). The group velocity of waves $\left(V_{\mathrm{g}}\right)$ decreases with an increase in the wave number $(k)$ for different values of $\kappa$ and $\mu$. It is very interesting to see that for smaller $k<0.5$ (i.e., for larger wavelength), the group velocity reduces with an increase in $\mu$; however, it increases with larger $k$. Furthermore, an increase in the parameter $\kappa$ (e.g., from $\kappa=4$ to $\kappa=8$ ) leads to an enhancement of $V_{\mathrm{g}}$ as $k \rightarrow 0$. Here, note that further increase of $\kappa(>8)$ does not give any significant change in $V_{\mathrm{g}}$.

For $l=0,1$ and 2, we can determine the second-order harmonic modes in terms of $\phi_{1}^{(1)}$. So, for $m=2, l=1$, we have the reduced equations 
Fig. 1 Carrier wave frequency $\omega$ (upper panel) and the group velocity of the wave packet $V_{\mathrm{g}}$ (lower panel) are plotted against the wave number $k$ for different values of $\kappa$ and $\mu$ as shown in the legends (a)

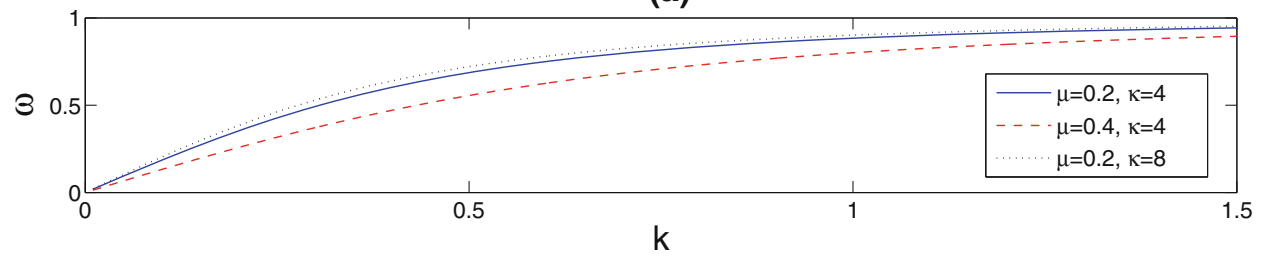

(b)

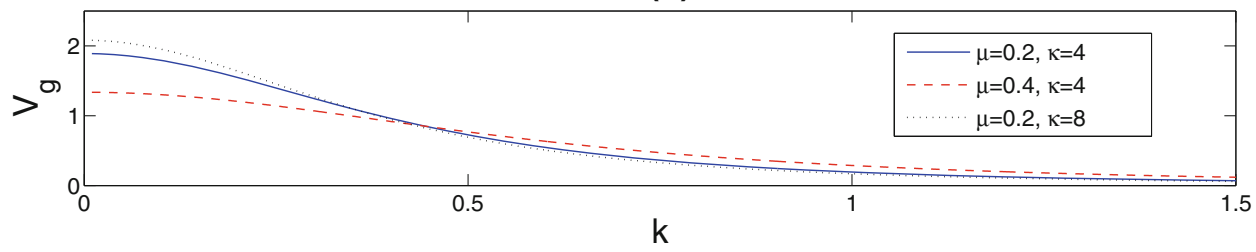

$i \omega n_{1}^{(2)}+i k w_{1}^{(2)}=V_{\mathrm{g}} \frac{\partial n_{1}^{(1)}}{\partial \zeta}$,

$i \omega w_{1}^{(2)}+i k \phi_{1}^{(2)}=V_{\mathrm{g}} \frac{\partial w_{1}^{(1)}}{\partial \zeta}-\frac{\partial \phi_{1}^{(1)}}{\partial \zeta}$,

with

$$
\begin{aligned}
& u_{1}^{(1)}=\frac{\omega_{\mathrm{c}} \frac{\partial \phi_{1}^{(1)}}{\partial \eta}-i \omega \frac{\partial \phi_{1}^{(1)}}{\partial \xi}}{\omega^{2}-\omega_{\mathrm{c}}^{2}}, \\
& v_{1}^{(1)}=-\left[\frac{i \omega \frac{\partial \phi_{1}^{(1)}}{\partial \eta}+\omega_{\mathrm{c}} \frac{\partial \phi_{1}^{(1)}}{\partial \xi}}{\omega^{2}-\omega_{\mathrm{c}}^{2}}\right] .
\end{aligned}
$$

The second-order harmonic modes with $m=2$ and $l=2$ are given by

$$
\begin{aligned}
& -\omega n_{2}^{(2)}+k w_{2}^{(2)}+k n_{1}^{(1)} w_{1}^{(1)}=0, \\
& -2 \omega w_{2}^{(2)}+k\left(w_{1}^{(1)}\right)^{2}+k \phi_{2}^{(2)}=0 .
\end{aligned}
$$

Thus, we obtain

$n_{2}^{(2)}=C_{1}^{(22)}\left(\phi_{1}^{(1)}\right)^{2}, u_{2}^{(2)}=C_{2}^{(22)}\left(\phi_{1}^{(1)}\right)^{2}$,

$\phi_{2}^{(2)}=C_{3}^{(22)}\left(\phi_{1}^{(1)}\right)^{2}$,

where the coefficients are

$$
\begin{aligned}
& C_{1}^{(22)}=\left(4 k^{2}+q_{1}\right) C_{3}^{(22)}+q_{2}, \\
& C_{2}^{(22)}=\frac{\omega}{k}\left[C_{1}^{(22)}-\left(k^{2}+q_{1}\right)^{2}\right], \\
& C_{3}^{(22)}=\frac{q_{2}}{3 k^{2}}+\frac{k^{2}}{2 \omega^{4}} .
\end{aligned}
$$

We note that the first-order zeroth harmonics $\left(n_{0}^{(1)}, w_{0}^{(1)}\right.$, $\phi_{0}^{(1)}$ ) vanish [35], which gives $u_{0}^{(1)}=v_{0}^{(1)}=0$. For $m=2$, $l=0$, we obtain the second-order and zeroth-order harmonic modes in the following forms:
$n_{0}^{(2)}=C_{1}^{(20)}\left(\phi_{1}^{(1)}\right)^{2}, u_{0}^{(2)}=C_{2}^{(20)}\left(\phi_{1}^{(1)}\right)^{2}$,

$\phi_{0}^{(2)}=C_{3}^{(20)}\left(\phi_{1}^{(1)}\right)^{2}$,

where the coefficients are

$$
\begin{aligned}
& C_{1}^{(20)}=q_{1} C_{3}^{(20)}+2 q_{2}, \\
& C_{2}^{(20)}=V_{\mathrm{g}} C_{1}^{(20)}-2 \frac{\omega}{k}\left(k^{2}+q_{1}\right)^{2}, \\
& C_{3}^{(20)}=\frac{2 q_{2} V_{\mathrm{g}}^{2}-\left(k^{2}+3 q_{1}\right)}{1-q_{1} V_{\mathrm{g}}^{2}} .
\end{aligned}
$$

Proceeding to the next order $(m=3)$ and solving for the first harmonic equations $(l=1)$, an explicit compatibility condition is determined, from which we obtain the following NLS equation for $\Phi \equiv \phi_{1}^{(1)}$ :

$i \frac{\partial \Phi}{\partial \tau}+P \frac{\partial^{2} \Phi}{\partial \zeta^{2}}+Q|\Phi|^{2} \Phi-S\left(\frac{\partial^{2} \Phi}{\partial \xi^{2}}+\frac{\partial^{2} \Phi}{\partial \eta^{2}}\right)=0$.

The coefficient of dispersion $P$ and the nonlinearity $Q$ are given by

$$
\begin{aligned}
P \equiv & \omega^{\prime \prime}(k)=-\frac{3}{2} q_{1} \frac{\omega^{5}}{k^{4}}, \\
Q= & \frac{\omega^{3}}{k^{2}}\left[\frac{3}{2} q_{3}+q_{2}\left\{C_{3}^{(20)}+C_{3}^{(22)}\right\}\right] \\
& -\frac{\omega}{2}\left\{C_{1}^{(20)}+C_{1}^{(22)}\right\}-k\left\{C_{2}^{(22)}+C_{2}^{(20)}\right\} .
\end{aligned}
$$

The coefficient $S$ which accounts for the combined effects of transverse perturbations and the external magnetic field is given by

$$
S=\frac{\omega^{3}}{2 k^{2}\left(\omega_{\mathrm{c}}^{2}-\omega^{2}\right)} \text {. }
$$




\section{Stability analysis}

We note that the amplitude modulation of DIA wave envelopes typically depend on the coefficients of the NLS equation (31), which parametrically depend on the density ratio $\mu$, the superthermality of electrons (via $\kappa$ ) as well as the intensity of the magnetic field (via $\omega_{\mathrm{c}}$ ). Inspecting the coefficients $P, Q$ and $S$, we find that $P=-(3 / 2) q_{1} \frac{\omega^{5}}{k^{4}} \equiv-(3 / 2) \frac{\omega^{3}}{k^{2}}\left(1-\omega^{2}\right)$, i.e., $P$ is always negative for $\kappa>3 / 2$ (for which $q_{1}>0$ ) and $\omega<1$. However, $Q$ can be positive or negative depending on the values of $k, \mu$ and $\kappa$. Also, $S>0(S<0)$ according to when $\omega<\omega_{\mathrm{c}}\left(\omega>\omega_{\mathrm{c}}\right)$. We will find that the key elements responsible for the MI are the ratios $P / Q$ and $S / P$ together with their signs and magnitudes. Considering a harmonic wave solution of Eq. (31) of the form $\Phi=$ $\Phi_{0} \exp \left(i Q\left|\Phi_{0}\right|^{2} \tau\right)$ with $\Phi_{0}$ denoting the constant amplitude, one can obtain the following dispersion relation for the modulated DIA wave packets [25]:

$$
\begin{aligned}
\Omega^{2}= & K^{4}\left(\frac{P \alpha^{2}-S}{1+\alpha^{2}}\right)^{2} \\
& \times\left(1-\frac{2\left(1+\alpha^{2}\right)\left|\Phi_{0}\right|^{2}}{K^{2}} \frac{Q / P}{\alpha^{2}-S / P}\right),
\end{aligned}
$$

where $\Omega$ and $K \equiv \sqrt{K_{\xi}^{2}+K_{\eta}^{2}+K_{\zeta}^{2}}$, respectively, denote the wave frequency and the wave number of modulation. The parameter $\alpha \equiv K_{\zeta} / \sqrt{K_{\xi}^{2}+K_{\eta}^{2}}$ is related to the modulational obliqueness $\theta$ which the wave vector $\mathbf{K}$ makes with the resultant of $K_{\xi} \hat{x}$ and $K_{\eta} \hat{y}$, i.e., $\theta=\arctan (\alpha)$. From Eq. (35), we find that there exists a critical wave number $K_{\mathrm{c}}$ such that $K^{2}<K_{\mathrm{c}}^{2} \equiv 2\left|\Phi_{0}\right|^{2}\left(1+\alpha^{2}\right)(Q / P) /\left(\alpha^{2}-S / P\right)$, the MI sets in either for $P Q>0, \alpha_{1}^{2}-S / P>0$ or for $P Q<0$, $\alpha_{1}^{2}-S / P<0$ [25]. It is further found that a critical value of $\theta$, i.e., $\theta_{\mathrm{c}} \equiv \arctan (\sqrt{S / P})$ also exists for the occurrence of $M I$. Thus, the MI may occur either for $P Q>0, \theta>\theta_{\mathrm{c}}$ or $P Q<0, \theta<\theta_{\text {c }}$, i.e., we have two possible cases:

- Case I: when $\omega<\omega_{\mathrm{c}}$, the MI sets in for $Q<0$ and for any value of $\theta$ in $0 \leq \theta \lesssim \pi / 2$.

- Case II: when $\omega>\omega_{\mathrm{c}}$, the MI sets in either for $Q<0$ and $\theta>\theta_{\mathrm{c}}$ or $Q>0$ and $\theta<\theta_{\mathrm{c}}$.

From the subsequent analysis and Fig. 2, it will be clear that the Case I is not admissible to the present study as there is no common region for which $\omega<\omega_{\mathrm{c}}$ and $Q<0$ are satisfied. So, we will focus only on Case II. It turns out that when the DIA wave frequency is larger than the ion-cyclotron frequency $\omega_{c}$, the MI is related to the obliqueness parameter $\theta$; however, the instability disappears for $\omega<\omega_{\mathrm{c}}$. We numerically investigate different stable and unstable regions in the $\omega-\theta$ plane as shown in Fig. 2. We find that the charged dust impurity (represented by the parameter $\mu$ with $0<\mu<1$ ), the superthermal parameter $\kappa$ and the gyrofrequency $\omega_{\mathrm{c}}$ shift the stable/unstable regions around the $\omega-\theta$ plane. From panels $(a, b)$ it is clear that as $\mu$ increases, i.e., as the number of charged dust grains decreases, a part of the instability region (with $\theta<\theta_{c}$ ) shifts to a stable one and the region of stability in the $\omega-\theta$ plane increases. However, the instability region with $\theta>\theta_{\mathrm{c}}$ increases slightly with increasing values of $\mu$. This implies that when the obliqueness parameter $\theta$ is below its critical value $\theta_{\mathrm{c}}$, the presence of charged dust impurity in the plasma favors the instability of modulated wave packets. Comparing panel (c) with panel (a) we find that the superthermality of electrons (with lower values of $\kappa$ ) also favors the instability in the region with $\theta<\theta_{\mathrm{c}}$. The instability region with $\theta>\theta_{\mathrm{c}}$ remains almost unchanged. From panels (a) and (d) it is also evident that the external magnetic field significantly reduces the regions of instability both in the cases of $\theta<\theta_{\mathrm{c}}$ and $\theta>\theta_{\mathrm{c}}$.

The maximum growth/decay rate $\Gamma_{\max }=\operatorname{Im}(\Omega)_{\max }$ can be obtained from Eq. (35) as $\Gamma_{\max }=Q\left|\phi_{0}\right|^{2}$ provided $P K_{\zeta}^{2}-S\left(K_{\xi}^{2}+K_{\eta}^{2}\right)=Q\left|\phi_{0}\right|^{2}$ is satisfied. The decay rate of MI is depicted in Fig. 3 for different values of $\kappa, \mu$ and $\omega_{\mathrm{c}}$. Clearly, the effects of higher values of $\kappa$ (less superthermality) suppresses the instability decay rate with cutoffs at significantly lower wave numbers of modulation (see the solid and dotted lines). However, the decay rate becomes higher with increasing values of the electron concentration (or decreasing the dust concentration) with cutoffs at higher $K$ (see the solid and dashed lines). We find that in contrast to the unmagnetized plasmas, the effect of $\omega_{\mathrm{c}}$ is to increase the cutoffs at higher wave numbers of modulation; however, the decay rate is slightly reduced (see the solid and dash-dotted lines).

\section{Summary and conclusion}

We have investigated the amplitude modulation of DIA wave packets in a magnetized multicomponent plasma consisting of singly charged positive ions, superthermal electrons featuring kappa distribution and negatively charged immobile dust grains. Using the multiple-scale technique, an NLS equation is derived which governs the evolution of DIA wave envelopes. It is shown that both the dispersive and the nonlinear coefficients of the NLS equation are significantly modified by the effects of charged dust impurity, the external magnetic field as well as the superthermality of electrons. Different stable and unstable regions under modulation are obtained in the plane of the carrier wave frequency $(\omega)$ and the obliqueness $(\theta)$ of modulation. It is found that the parameters $\kappa, \mu$, 
(a)

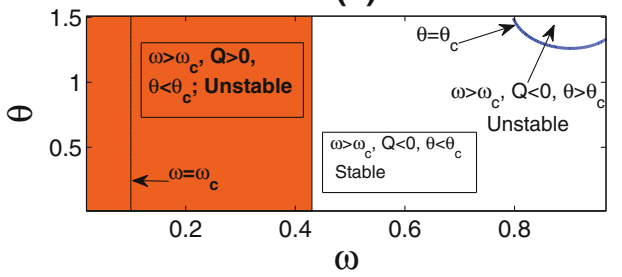

(c)

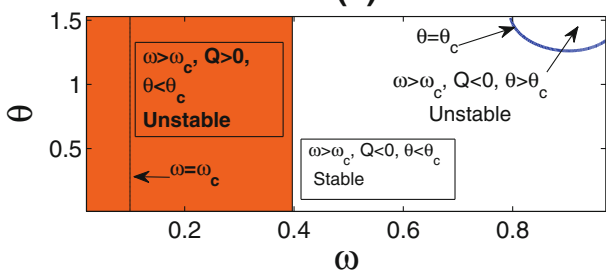

Fig. 2 The stable and unstable regions of wave modulation are shown by the contour plots of $Q=0, \omega=\omega_{\mathrm{c}}$ and $\theta=\theta_{\mathrm{c}}$ in the $\omega-\theta$ plane for different values of the parameters: $\mathbf{a} \kappa=4, \omega_{\mathrm{c}}=0.1$ and $\mu=0.2$, b $\kappa=4, \omega_{\mathrm{c}}=0.1$ and $\mu=0.3$, c $\kappa=6, \omega_{\mathrm{c}}=0.1$ and $\mu=0.2$, and $\mathbf{d}$ $\kappa=4, \omega_{\mathrm{c}}=0.3$ and $\mu=0.2$. The shaded or gray (blank or white) (b)

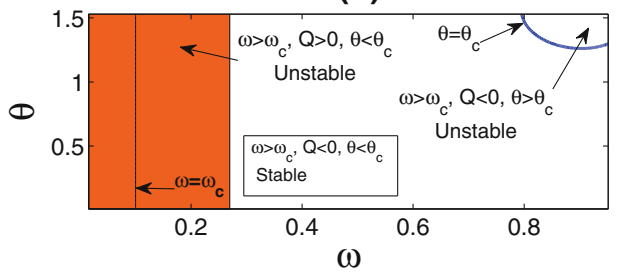

(d)

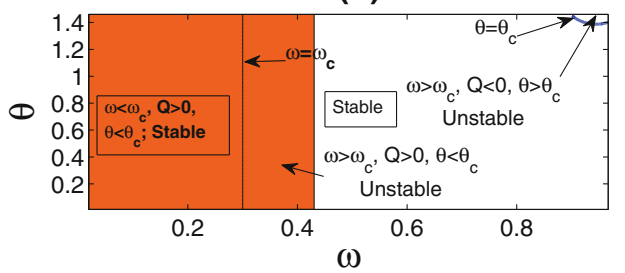

region stands for $Q>0(Q<0)$. When $\omega>\omega_{\mathrm{c}}$, the $M I$ occurs either for $Q>0, \theta<\theta_{\mathrm{c}}$, or for $Q<0, \theta>\theta_{\mathrm{c}}$. No instability occurs in the regime $\omega<\omega_{\mathrm{c}}$. In some other regions the modulated wave becomes stable
Fig. 3 The decay rate of modulational instability $\Gamma$ is shown against the wave number of modulation $K$ with the variation of the parameters as shown in the legend. The other parameter values are $k=0.5$ and $\phi_{0}=0.5$

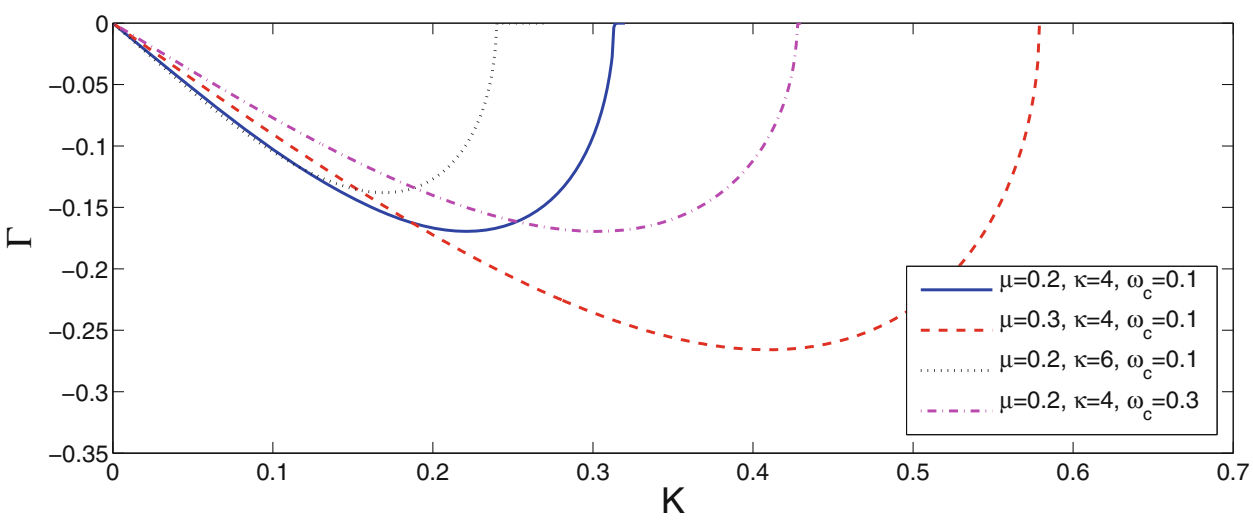

$\omega_{\mathrm{c}}$ and $\theta$ remarkably shift the stable/unstable regions around the $\omega-\theta$ plane. The growth/decay rate of instability is also examined numerically with these plasma parameters. The main results are summarized as follows:

- Starting from a set of fluid equations, the dynamics of weakly nonlinear, slowly varying DIA wave packets is shown to be governed by a three-dimensional NLS equation in which the additional dispersive terms (leading to two more space dimensions in the equation) appear due to the combined effects of the transverse perturbations and the external magnetic field. The fluid model with $\kappa$-distributed electrons and stationary charged dust particles is valid for the plasma parameters satisfying $0<\mu<1$ and $\kappa>3 / 2$.

- The carrier wave frequency is seen to assume a constant value at large $k>1$, and approaches the ion plasma frequency with increasing values of $\kappa$. The wave frequency $\omega$ and hence the group velocity $V_{\mathrm{g}}$ get significantly reduced with higher values of $\mu$.

- The group velocity dispersion $P$ of the NLS equation is always negative irrespective of the values of $k$ and the plasma parameters. The nonlinear coefficient $Q$ is always negative for $k \gtrsim 1$; however, it can be either positive or negative in the range $0<k<1$ depending on the values of $\kappa$ and $\mu$. For propagation below the ion cyclotron frequency, the DIA wave packet is always stable. However, for $\omega>\omega_{\mathrm{c}}$, it is unstable and the MI is related to the obliqueness parameter $\theta$. The parameters $\kappa, \mu$ and $\omega_{\mathrm{c}}$ are found to shift the instability regions around the $\omega-\theta$ plane significantly.

- The decay rate of MI is found to be significantly suppressed by the effects of $\kappa$, i.e., when $\kappa$ increases with cutoffs at lower wave numbers of modulation. However, it can be higher with increasing values of the density ratio $\mu$. The effect of the external magnetic field 
is to decrease the decay rate with cutoffs at higher values of the wave number of modulation.

The findings of the present investigation may be useful for the modulation of dust-ion acoustic wave envelopes in dusty superthermal plasmas such as those in laboratory [36], space [37-39] and astrophysical [40] environments.

Acknowledgements This work was supported by DRS-II(SAP) no. F 530/17/DRS-II/2015(SAP-I) UGC, New Delhi. A. P. M. acknowledges support from UGC-SAP (DRS, Phase III) with Sanction order no. F.510/3/DRS-III/2015(SAPI) dated 25/03/2015, and UGC-MRP with F. no. 43-539/2014 (SR) and FD Diary no. 3668 dated 17.09.2015.

Open Access This article is distributed under the terms of the Creative Commons Attribution 4.0 International License (http://crea tivecommons.org/licenses/by/4.0/), which permits unrestricted use, distribution, and reproduction in any medium, provided you give appropriate credit to the original author(s) and the source, provide a link to the Creative Commons license, and indicate if changes were made.

\section{References}

1. Shukla, P.K., Mendis, D.A., Desai, T.: Advances in dusty plasmas. World Scientific, Singapore (1999)

2. Shukla, P.K., Mamun, A.A.: Introduction to dusty plasma physics. Institute of Physics, Bristol (2002)

3. Boufendi, L., Mikikian, M., Shukla, P.K.: New vistas in dusty plasmas. In: AIP Proceeding, AIP, New York (2005)

4. Shukla, P.K., Silin, V.P.: Dust ion-acoustic wave. Phys. Scrip. 45, 508 (1992)

5. Barkan, A., D'Angelo, N., Merlino, R.: Experiments on ionacoustic waves in dusty plasmas. Planet. Space. Sci. 44, 239 (1996)

6. Summers, D., Thorne, R.M.: The modified plasma dispersion function. Phys. Fluids B 3, 1835 (1991)

7. Sittler Jr., E.C., Ogilvie, K.W., Scudder, J.D.: Survey of lowenergy plasma electrons in Saturn's magnetosphere: Voyagers 1 and 2. J. Geophys. Res. 88, 8847 (1983)

8. Mace, R.L., Hellberg, M.A.: A dispersion function for plasmas containing superthermal particles. Phys. Plasmas 2, 2098 (1995)

9. Vasyliunas, V.M.: A survey of low-energy electrons in the evening sector of the magnetosphere with OGO 1 and OGO 3. J. Geophys. Res. 73, 2839 (1968)

10. Hellberg, M.A., Mace, R.L., Armstrong, R.J., Karlstad, G.: Electron-acoustic waves in the laboratory: an experiment revisited. J. Plasma Phys. 64, 433 (2000)

11. Saini, N.S., Kourakis, I., Hellberg, M.A.: Arbitrary amplitude ion-acoustic solitary excitations in the presence of excess superthermal electrons. Phys. Plasmas 16, 062903 (2009)

12. Shah, A., Mahmood, S., Haque, Q.: Propagation of solitary waves in relativistic electron-positron-ion plasmas with kappa distributed electrons and positrons. Phys. Plasmas 18, 114501 (2011)

13. El-Tantawy, S.A., El-Bedwehy, N.A., Moslem, W.M.: Nonlinear ion-acoustic structures in dusty plasma with superthermal electrons and positrons. Phys. Plasmas 18, 052113 (2011)

14. Sultana, S., Kourakis, I.: Electrostatic solitary waves in the presence of excess superthermal electrons: modulational instability and envelope soliton modes. Plasma Phys. Control. Fusion 53, 045003 (2011)
15. Shahmansouri, M., Tribeche, M.: Propagation properties of ion acoustic waves in a magnetized superthermal bi-ion plasma. Astrophys. Space Sci. 350, 623 (2014)

16. Adnan, M., Mahmood, S., Qamar, A.: Small amplitude ion acoustic solitons in a weakly magnetized plasma with anisotropic ion pressure and kappa distributed electrons. Adv. Sp. Res. 53, 845 (2014)

17. Shahmansouri, M., Astaraki, E.: Transverse perturbation on three-dimensional ion acoustic waves in electron-positron-ion plasma with high-energy tail electron and positron distribution. J. Theor. Appl. Phys. 8, 189 (2014)

18. Shalini K, Saini N.S., Misra A.P.: Modulation of ion-acoustic waves in a nonextensive plasma with two-temperature electrons. Phys. Plasmas. 22, 092124 (2015)

19. Kourakis, I., Shukla, P.K.: Ion-acoustic waves in a two-electrontemperature plasma: oblique modulation and envelope excitations. J. Phys. A Math. Gen. 36, 11901 (2003)

20. Kourakis, I., Shukla, P.K.: Electron-acoustic plasma waves: oblique modulation and envelope solitons. Phys. Rev. E. 69, 036411 (2004)

21. Kourakis, I., Shukla, P.K.: Finite ion temperature effects on oblique modulational stability and envelope excitations of dustion acoustic waves. Euro. Phys. J. D. 28, 109 (2004)

22. Kourakis, I., Shukla, P.K.: Oblique amplitude modulation of dustacoustic plasma waves. Phys. Scr. 69, 316 (2004)

23. Misra, A.P., Bhowmik, C.: Nonlinear wave modulation in a quantum magnetoplasma. Phys. Plasmas 14, 012309 (2007)

24. Saini, N.S., Kourakis, I.: Dust-acoustic wave modulation in the presence of superthermal ions. Phys. Plasmas 15, 123701 (2008)

25. Bains, A.S., Misra, A.P., Saini, N.S., Gill, T.S.: Modulational instability of ion-acoustic wave envelopes in magnetized quantum electron-positron-ion plasmas. Phys. Plasmas 17, 012103 (2010)

26. El-Taibany, W.F., Kourakis, I.: Modulational instability of dust acoustic waves in dusty plasmas: modulation obliqueness, background ion nonthermality, and dust charging effects. Phys. Plasmas 13, 062302 (2006)

27. El-Labany, S.K., El-Shewy, E.K., Abd El-Razek, H.N., El-Rahman, A.A.: Wave propagation in strongly dispersive superthermal dusty plasma. Adv. Sp. Res. 59, 1962 (2017)

28. Ahmadihojatabad, N., Abbasi, H., Pajouh, H.H.: Influence of superthermal and trapped electrons on oblique propagation of ion-acoustic waves in magnetized plasma. Phys. Plasmas 17, $112305(2010)$

29. Guo, S., Mei, L.: Three-dimensional dust-ion-acoustic rogue waves in a magnetized dusty pair-ion plasma with nonthermal nonextensive electrons and opposite polarity dust grains. Phys. Plasmas 21, 82303 (2014)

30. Gharaee, H., Afghah, S., Abbasi, H.: Modulational instability of ion-acoustic waves in plasmas with superthermal electrons. Phys. Plasmas 18, 032116 (2011)

31. Hellberg, M.A., Mace, R.L., Baluku, T.K., Kourakis, I., Saini, N.S.: Comment on "Mathematical and physical aspects of Kappa velocity distribution". Phys. Plasmas 16, 094701 (2009)

32. Taniuti, T., Yajima, N.: Perturbation method for a nonlinear wave modulation. J. Math. Phys. 10, 1369 (1969)

33. Asano, N., Taniuti, T., Yajima, N.: Perturbation method for a nonlinear wave modulation. J. Math. Phys. 10, 2020 (1969)

34. Xue, J-K: Modulation of magnetized multidimensional waves in dusty plasma. Phys. Plasmas 12, 062313 (2005)

35. Taniuti, T.: Reductive perturbation method and far fields of wave equations. Progress Theoret. Phys. Suppl. 55, 1 (1974)

36. Liu, J.M., DeGroot, J.S., Matte, J.P., Johnston, T.W., Drake, R.P.: Measurements of inverse bremsstrahlung absorption and nonmaxwellian electron velocity distributions. Phys. Rev. Lett. 72, 2717 (1994) 
37. Montgomery, M.D., Bame, S.J., Hundhausen, A.J.: Solar wind electrons: vela 4 measurements. J. Geophys. Res. 73, 4999 (1968)

38. Maksimovic, M., Pierrard, V., Riley, P.: A kinetic model of the solar wind with Kappa distribution functions in the corona. Geophys. Res. Lett. 24, 1151 (1997)

39. Zouganelis, I.: Measuring suprathermal electron parameters in space plasmas: implementation of the quasi-thermal noise spectroscopy with kappa distributions using in situ Ulysses/ URAP radio measurements in the solar wind. J. Geophys. Res. 113, A08111 (2008)

40. Pierrard, V., Lazar, M.: Kappa distributions: theory and applications in space plasmas. Solar Phys. 267, 153 (2010). (and references therein) 